\title{
Effect of CXCR4 on Apoptosis in Osteosarcoma Cells via the PI3K/Akt/NF-k $\beta$ Signaling Pathway
}

\author{
Chunming Jiang ${ }^{a}$ Shenglin Ma ${ }^{b}$ Runlei Hu${ }^{c}$ Xuepeng Wang ${ }^{\mathrm{d}, \mathrm{e}}$ Maoqiang Lid,e \\ Fei Tiand,e Wu Jiang d,e Liulong Zhu ${ }^{\text {d,e }}$ Zhenyu Bian ${ }^{d, e}$ \\ aDepartment of Pediatrics, Hangzhou First People's Hospital, Nanjing Medical University, Hangzhou, \\ Zhejiang, 'Department of Radiation Oncology, Hangzhou First People's Hospital, Nanjing Medical \\ University, Hangzhou, Zhejiang, 'Department of Thoracic Surgery, Hangzhou First People's Hospital, \\ Nanjing Medical University, Hangzhou, Zhejiang, 'Department of Orthopedic Surgery, Hangzhou First \\ People's Hospital, Nanjing Medical University, Hangzhou, Zhejiang, eHangzhou Orthopedic Institute, \\ Hangzhou, China
}

\section{Key Words}

Osteosarcoma $•$ CXCR4 $•$ Apoptosis $・ P I 3 K / A k t / N F-K \beta$ signaling $・$ ERS

\section{Abstract}

Background/Aims: Osteosarcoma, the most common primary bone malignancy, arises from primitive transformed cells of mesenchymal origin with the worldwide increasing morbidity and mortality. Previous studies found apoptosis of osteosarcoma cells was essential for an effective manner to improve the progress of osteosarcoma, and CXCR4 has been demonstrated to be relevant with various tumor progress and metastasis. Methods: The proliferation of cells transfected with CXCR4 shRNA and control shRNA were measured by BrdU assay. Apoptosis was detected by flow cytometry. Apoptotic protein expression levels were detected by Western blot. Caspase activity was detected by Colorimetric Assay Kits using microplate reader. Activation of NF-K $\beta$ signaling after CXCR4 down-regulation in osteosarcoma cells was examined by constructing NF-K $\beta$ promoter luciferase reporter plasmid. The expression and activation of NF$\kappa \beta$ Signaling relevant protein were analyzed to investigate the relationship between Akt and NF-K $\beta$ signaling after the down-regulation of CXCR4 in osteosarcoma cells. Results: Downregulation of CXCR4 significantly reduced the cell proliferation, while remarkably increased the cell apoptosis and apoptotic protein expression levels in osteosarcoma cells. Furthermore, down-regulation of CXCR4 induced cell apoptosis was caspase dependent in osteosarcoma cells. This study also showed CXCR4 down-regulation induced apoptosis through inhibiting PI3K/Akt/NF-K $\beta$ signaling pathway. In addition, endoplasmic reticulum stress (ERS) activation was involved in cell apoptosis induced down-regulation of CXCR4. Knockdown of partial ERS relevant proteins followed down-regulation of CXCR4 significantly inhibited cell apoptosis and the apoptotic protein expression levels. Conclusions: Taken together, the results demonstrated that down-regulation of CXCR4 could induce apoptosis of human osteosarcoma cells through inhibiting PI3K/Akt/NF-K $\beta$ signaling pathway, indicating that CXCR4 could be vital for the clinical therapy of osteosarcoma.

Zhenyu Bian and Liulong Zhu
Department of Orthopedic Surgery, Hangzhou First People's Hospital, Nanjing Medical University, 261 Huansha Road, Hangzhou, Zhejiang (China)

E-Mail zhenyub1@163.com / zhuliulong6@163.com 


\section{Cellular Physiology Cell Physiol Biochem 2018;46:2250-2260 \\ \begin{tabular}{l|l} 
DOI: 10.1159/000489593 & and Biochemistry \\
Published online:TMay 01, 2018 & $\begin{array}{l}\text { O 2018 The Author(s). Published by S. Karger AG, Basel } \\
\text { www.karger.com/cpb }\end{array}$
\end{tabular} \\ Jiang et al.: Mechanism of CXCR4 on Osteosarcoma}

\section{Introduction}

Osteosarcoma, arising from primitive transformed cells of mesenchymal origin, is the most common primary bone cancer, which exhibits osteoblastic differentiation and produces malignant osteoid $[1,2]$. Osteosarcoma has a high incidence of one to three per million every year in children and adolescents [3,4]. Currently, the 5-year survival rate of osteosarcoma patients is quite low, especially in the patients insensitive to chemotherapy [4-6]. Although new targeting drugs have been developed in these years, side effects and poor response still disturb the osteosarcoma patients $[7,8]$. Therefore, it is necessary to found new and effective strategies to improve osteosarcoma therapy.

It's well known that the induction of apoptosis in critical in the therapy of tumor [9]. The apoptotic process consists of extrinsic and intrinsic factors induced caspase-dependent classical apoptosis, which is regulated by transmembrane receptors and mitochondrial pathway respectively $[10,11]$. Previous study found apoptosis of osteosarcoma cells was essential to improve the treatment of osteosarcoma [12]. NF- $\kappa \beta$ is recognized as a crucial mediator in many steps of cancer initiation and progression, including the interaction of immune system, inflammation and down-regulation of apoptosis $[13,14]$. IkBa could suppress the activation of NF- $\kappa \beta$ through binding to the dimmers of NF-kB, whereas IKKa largely enhanced the activation of NF- $\kappa \beta[15,16]$. In addition, phosphorylation of Akt, the downstream effector of PI3K, has been observed as a critical mediator in the NF- $\kappa \beta$ signaling pathway promoting cell proliferation and protecting cancer cells against apoptosis in cancer cells [17]. Previous studies demonstrated that PI3K/Akt/NF- $\kappa \beta$ signaling pathway was a crucial target in the mechanism investigation of therapeutic route in various cancer cells [18-20]. However, the detailed mechanisms of PI3K/Akt/NF- $\kappa \beta$ signaling pathway and with the relationship to apoptosis of cancer are still unknown.

CXCR4, also known as fusin, is a $45 \mathrm{kDa}$ rhodopsin-like G protein-linked CXC chemokine receptor, mainly expressed in blood and tissue cells, including dendritic cells, endothelial cells, astrocytes, and lymphocytes [21]. CXCR4 is the receptor of chemokine SDF-1, which regulates cell migration and involved in cancer progress and immunodeficiency disorder diseases $[22,23]$. Recent study reported that CXCR4 was involved in metastatic potential in osteosarcoma at bone marrow and the lungs [24]. Previous studies showed that cancer cells have higher CXCR4 expression levels compared to normal cells [25, 26]. In addition, over-expression of CXCR4 has been demonstrated to promote metastasis and associate with unfavorable prognosis in breast and lung cancer $[27,28]$. Although CXCR4 has been reported to be relevant with various tumor progress and metastasis [29], the detailed molecular mechanisms of CXCR4 on osteosarcoma were still obscure.

This study aims to investigate a novel potential therapy strategy of osteosarcoma. We tested whether CXCR4 can be used as a target for osteosarcoma therapy. Here, we demonstrated that the protein expression of CXCR4 significantly increased in osteosarcoma cells and down-regulation of CXCR4 induced cell apoptosis in osteosarcoma cells. This study also showed CXCR4 down-regulation induced apoptosis through the inhibition of PI3K/ Akt/NF- $\kappa \beta$ signaling, which involved in endoplasmic reticulum stress (ERS) activation in osteosarcoma cells. In addition, Knockdown of ERS related proteins significantly inhibited cell apoptosis and the apoptotic protein expression levels induced by the down-regulation of CXCR4. These results provide CXCR4 as a novel target of potential therapy in osteosarcoma.

\section{Materials and Methods}

\section{Medicines and regents}

LY294002 was acquired from Cell Signaling Technology (Beverly, MA, USA). The broad-spectrum caspase inhibitor (z-VAD-fmk) was acquired from Millipore (Billerica, MA, USA). Caspase-8 specific inhibitor (z-IETD-fmk) was obtained from BioVision (Mountain View, CA, USA). Caspase-9 specific inhibitor (z-LEHDfmk) was obtained from BioVision (Mountain View, CA, USA). 


\section{Cellular Physiology Cell Physiol Biochem 2018;46:2250-2260 \\ and Biochemistry Published onIme: Vay 07, $2018 \quad \begin{aligned} & \text { DOI: 10.1159/000489593 } 2018 \text { The Author(s). Published by S. Karger AG, Basel } \\ & \text { www.karger.com/cpb }\end{aligned}$}

Jiang et al.: Mechanism of CXCR4 on Osteosarcoma

Cell culture

The human osteosarcoma cell line MG63, Saos2, HOS, U2OS and Human Osteoblasts Hob were obtained from Shanghai Institute of Chinese Academy of Sciences (China). Cells were maintained in RPMI1640 (Gibco, USA) supplemented with $100 \mathrm{U} / \mathrm{mL}$ penicillin, $100 \mu \mathrm{g} / \mathrm{mL}$ streptomycin and 10\% fetal bovine serum at $37^{\circ} \mathrm{C}$ in a humid incubator with $5 \% \mathrm{CO}_{2}$.

\section{Proliferation Assays}

The BrdU assay was used to detect cell proliferation. Cells were plated and treated with control or CXCR4 shRNA. CXCR4 shRNA: 5'-GATCCTGCCCACCATCTACTCCATTTCAAGAGAATGGA GTAGATGGTGGGCATTTTTGGAAGCTAGCA-3'; Control shRNA: 5'-GATCCAATCGCATAGC GTATGCCGTTTTCAAGAGAAACGGCATACGCTATGCGATTTTTTTGGAAGCTAGCA-3'. BrdU (100 $\mu$ M) was added and incubated for $2 \mathrm{~h}$ at $37^{\circ} \mathrm{C}$. The cells were then fixed and incubated for $30 \mathrm{~min}$ at $37^{\circ} \mathrm{C}$. Cells were washed with PBS and incubated with BrdU antibody conjugated with peroxidase for $60 \mathrm{~min}$ at $37^{\circ} \mathrm{C}$. Substrate solution was added and the absorbance was measured at $370 \mathrm{~nm}$ with a microplate reader (Bio-Rad, Japan).

\section{Apoptosis assay}

The apoptosis detection kit (Biolegend, CA, USA) was used to detect apoptosis assay. Cells were incubated with indicated reagents, following treated with $5 \mu \mathrm{g} / \mathrm{ml}$ 7-aminoactinomycin D (7-AAD) and 2.5 $\mu \mathrm{g} / \mathrm{ml}$ Annexin V-fluorescein isothiocyanate (FITC), and the results were analyzed by flow cytometry on the FACSCalibur (BD Biosciences, CA, USA).

RNA extraction and quantitative real-time polymerase chain reaction analysis

TRIzol reagent (Invitrogen, Carlsbad, USA) was used to acquire Total RNA. Reverse-transcribed complementary DNA was synthesized using the Prime-Script® RT Reagent Kit (TaKaRa, Tokyo, Japan). qPCR analyses were using with LightCycler 480 SYBR Green I Master (Roche, Welwyn Garden, Swiss). CXCR4 forward primer (5'-CACTTCAGATAACTACACCG-3'); reverse primer (5'-ATCCAGACGCCAACATAGAC-3'), GAPDH: forward primer (5'-AATC CCATCACCATCTTCCA-3'); reverse primer (5'-TGGACTCCACGACGTACTCA $\left.-3^{\prime}\right)$.

\section{Western blot}

Protein was separated on SDS-polyacrylamide gels and transferred to a nitrocellulose membrane (Bio-Rad, Hercules, USA). The membrane probed with antibodies against CXCR4, p-AKT, Bcl-2, BAX, PARP, caspase-3, caspase-8, caspase-9, DR5, Bid, IKKa, IkBa, CHOP, GRP78, XBP-1, P-eIF2a, eIF2a, GAPDH were purchased from Cell Signaling Technology, antibodies against P50, P52, P65, c-rel, relB were purchased from Santa Cruz Biotechnology. The membrane was then probed with horseradish peroxidase-conjugating (HRP) secondary antibody (1:10000; GE Healthcare, Tokyo, Japan).

Caspase activity

Caspase-3, Caspase-8 and Caspase-9 Colorimetric Assay Kits (Biovision, CA, USA) were used to detect the Caspase-3, -8 and -9 activities, respectively. The absorbance was detected at $405 \mathrm{~nm}$ according to the manufacturer's protocol by using MR7000 microplate reader.

\section{Luciferase Reporter assays}

The promoter of NF- $\kappa \beta$ was amplified and cloned in pGL 3.0 luciferase reporter plasmid. The cells were seeded and transfected with pRL-CMV renilla luciferase reporter and the pGL 3.0 luciferase reporter plasmid. The activities of firefly and Renilla luciferases were detected using a dual luciferase reporter assay system (Promega).

Flow cytometric analysis

To detect CXCR4 expression, cells were incubated in 6-well plates. After treatment with CXCR4 shRNA or control, cells were harvested and stained with CXCR4-PE (ebioscience) at $4^{\circ} \mathrm{C}$ for $30 \mathrm{~min}$. Cells were analyzed with a flow cytometry (FACScan®; BD Biosciences). 
Adenovirus vector construction, infection and transfection

Oligonucleotides of shRNA for CRCX4, CHOP, GRP78, XBP-1, P-eIF2a were obtained from Sangon Biotech (Shanghai). The sequences of Akt, IKKa and IkBa were cloned into shuttle vector. HEK-293T cells were cultured in dish, attached to the plate for $12 \mathrm{~h}$. The linearized DNA, the shuttle vector and pacAd vector were cotransfected in cells by Lipofectamine 2000 (Invitrogen). Viral vectors were harvested, purified and tittered. The acquired adenovirus vectors were used to infect the cells.

\section{Statistical analysis}

Results are showed as the means \pm S.D. from at least three independent experiments. The statistical differences were calculated by the Student's $t$-test or one-way analysis of variance (ANOVA) with Dunnett's test.

\section{Results}

$\begin{array}{llr}\text { Role of } & \text { CXCR4 } & \text { in } \\ \text { osteosarcoma } & & \text { cells }\end{array}$ proliferation and apoptosis

The expression of CXCR4 was detected in osteosarcoma and Hob cells. As shown in Fig. 1a, the protein expression of CXCR4 in various osteosarcoma cells was significantly higher than Hob cells. Consistently, Higher mRNA level of CXCR4 was found in osteosarcoma cells compared with in Hob cells (Fig. 1b). To investigate the role of CXCR4 in osteosarcoma cells, we used the CXCR4 shRNA adenovirus to infect osteosarcoma cells, and the expression of CXCR4 was markedly decreased compared with control (Fig. 1c). Furthermore, CXCR4 shRNA also decreased CXCR4 expression on the membrane surface (Fig. 1i). The effect of CXCR4 shRNA on osteosarcoma cell proliferation was determined by BrdU assay. The proliferation of cell was significant decreased in a time-dependent manner with the CXCR4 shRNA treatment (Fig. 1f). We also performed apoptosis assay using double stained with 7AAD and Annexin $\mathrm{V}$ in osteosarcoma cell treated with CXCR4 shRNA. As shown in

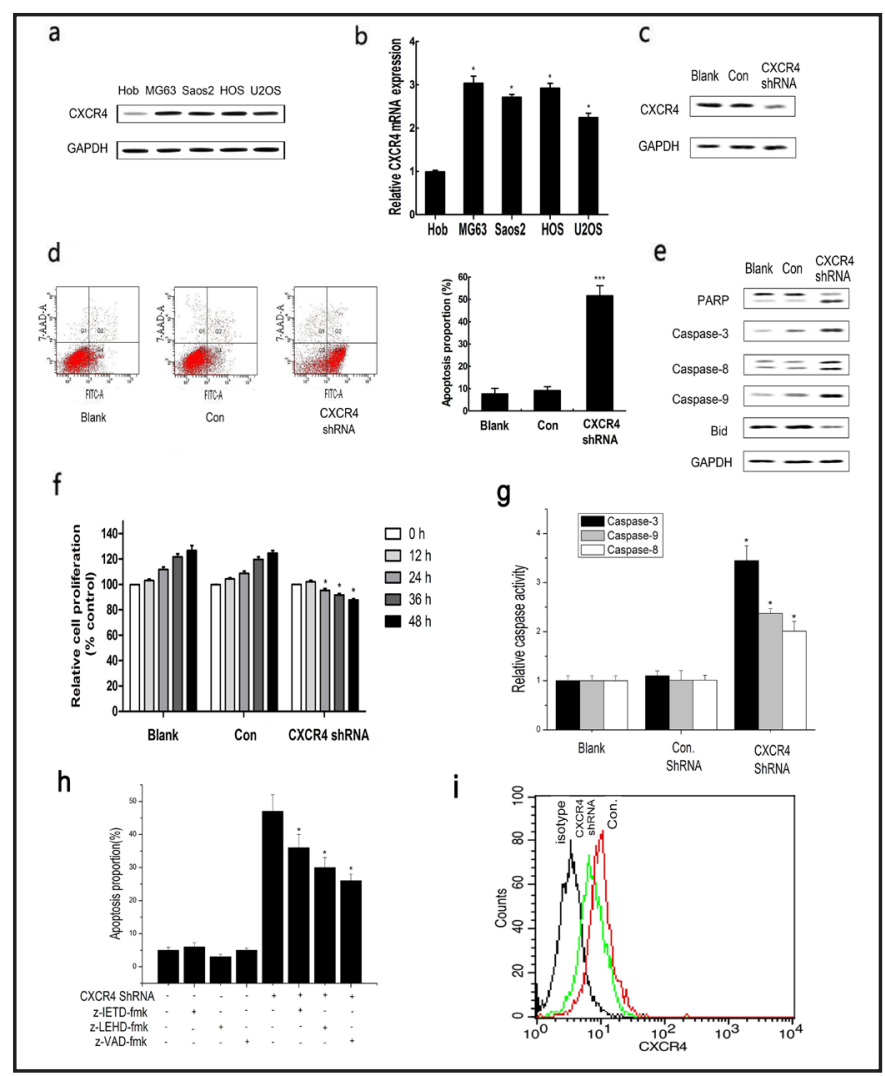

Fig. 1. CXCR4 down-regulation induced cell apoptosis in osteosarcoma cells. (a) The protein expression of CXCR4 was detected by Western Blot in osteosarcoma and Hob cells. (b) CXCR4 mRNA was measured by Real-time PCR analysis in osteosarcoma and Hob cells. ${ }^{*} \mathrm{P}<0.05$. (c) The protein expression of CXCR4 was detected in MG63 osteosarcoma cells following treatment with CXCR4 shRNA or control shRNA for $24 \mathrm{~h}$. (d) Cells were treated with CXCR4 shRNA or control shRNA for $48 \mathrm{~h}$. Cell apoptosis was detected with annexin V-FITC/7-AAD and analyzed by flow cytometry. ${ }^{* * *} \mathrm{P}<0.001$ VS Blank. (e) Western blotting analysis for protein expressions of PARP, caspase-3, 8, 9 and Bid were measured in osteosarcoma cells after down-regulation with CXCR4. (f) The proliferative effect of CXCR 4 on osteosarcoma cells. ${ }^{*} \mathrm{P}<0.05$ VS Blank. (g) Caspase activities in osteosarcoma cells. ${ }^{*} \mathrm{P}<0.05$ VS Blank (h) Cell apoptosis was detected for osteosarcoma cells treated with CRCX4 shRNA followed by the pretreatment with caspase inhibitors for $1 \mathrm{~h} .{ }^{*} \mathrm{P}<0.05$ VS CXCR4 shRNA treatment. (i) The expression of CXCR4 was detected by flow cytometry after treatment with CXCR4 or control shRNA in MG63 osteosarcoma cells. 
Fig. 2. CXCR4 down-regulation induced Akt and NF- $K B$ inhibition in osteosarcoma cells. (a) The protein expression of p-AKT was detected in MG63 osteosarcoma cells after CXCR4 down-regulation. (b) The protein expression of p-AKT was detected in osteosarcoma cells after treatment with CXCR4 down-regulation and/or Akt overexpression. (c) The protein expression of p-AKT was detected in osteosarcoma cells after treatment with CXCR4 down-regulation and/ or LY294002. (d) Cell apoptosis was detected for osteosarcoma cells treated with CXCR4 shRNA followed transfection with Akt or LY294002. (e) CXCR4 shRNA decreased the transcriptional activity of NF-KB promoter. After incubating with or without CXCR4 shRNA, MG63 cells were prepared for luciferase assays. Values were normalized as the arbitrary luciferase activity. (f) The protein expressions of P50, P52, P65, c-rel, relB were detected in MG63 osteosarcoma cells after CXCR4 down-regulation. (g) The protein expression of IKKa was

a

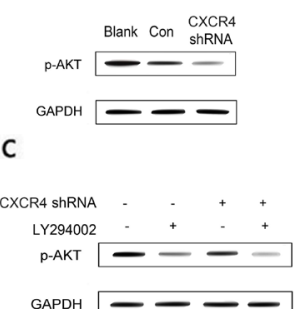

e

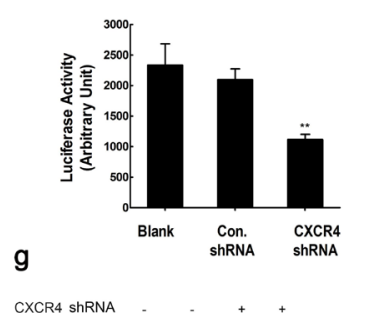

CXCR4 ShRNA

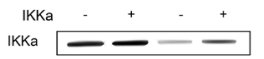

GAPDH ---

$\mathrm{h}$

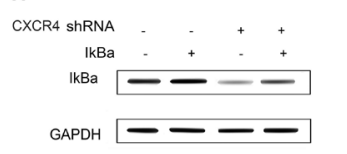

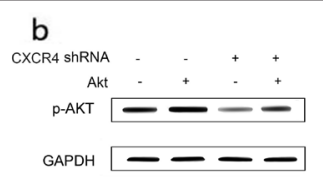

d

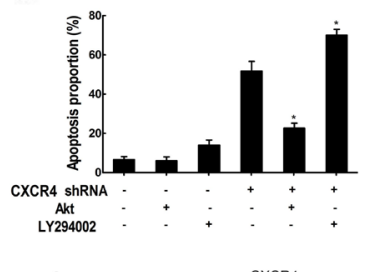

f

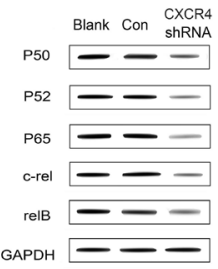

i

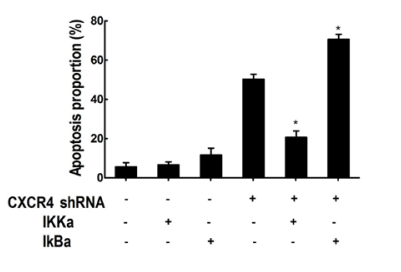
detected in MG63 osteosarcoma cells after treatment with CXCR4 down-regulation and/or IKKa overexpression. (h) The protein expression of IkBa was detected in MG63 osteosarcoma cells after treatment with CXCR4 down-regulation and/or IkBa overexpression. (i) Cell apoptosis was detected for osteosarcoma cells treated with CXCR4 shRNA followed transfection with IKKa or IkBa. ${ }^{*} \mathrm{P}<0.05$ VS CXCR4 shRNA treatment, ${ }^{* *} \mathrm{P}<0.01$ VS Blank.

Fig. 1d, apoptotic cells were significantly increased with CXCR4 shRNA treatment $(\mathrm{P}<0.001)$. Moreover, apoptosis related proteins,including PARP, caspase-3, caspase- 8 , caspase- 9 and Bid, were detected by western blot. The expression levels of PARP, caspase-3, caspase-8, caspase- 9 were obviously increased after CXCR4 shRNA treatment, whereas the expression level of Bid was expectedly decreased with CXCR4 shRNA treatment in osteosarcoma cells (Fig. 1e). Furthermore, we detected caspase activities of caspase-3, -8, and 9 after CXCR4 shRNA treatment. The caspase activities of caspase-3, -8, and 9 were markedly enhanced with CXCR4 shRNA treatment (Fig 1g). To further confirm these results, caspase inhibitors such as z-VAD-fmk, z-IETD-fmk and z-LEHD-fmk were used to pretreat osteosarcoma cells followed treatment with CXCR4 shRNA. Apoptosis induced by CXCR4 shRNA treatment was significantly decreased with z-IETD-fmk, z-LEHD-fmk or z-VAD-fmk pretreatments (Fig. 1h).

\section{CXCR4 down-regulation inhibited Akt activation in osteosarcoma cells}

To investigate the effect of CXCR4 on survival signaling in osteosarcoma cells, we analyzed the effect of CXCR4 shRNA on AKT activation. Treatment of osteosarcoma cells with CXCR4 shRNA could markedly reduce P-AKT protein expression compared with control (Fig. 2a). We further investigated the effect of Akt signaling in CXCR4 down-regulation induced apoptosis using Akt over-expression and PI3K-AKT inhibitor LY294002 (Fig. $2 \mathrm{~b}$ ). The p-AKT protein expression was obviously enhanced in the treatment with CXCR4 shRNA and Akt overexpression compared with CXCR4 shRNA treatment alone. In addition, 
Fig. 3. Relationship between Akt and NF- $\kappa b$ signaling followed CXCR4 down-regulation in osteosarcoma cells. (a, c) The protein expressions of P50, P52, P65, c-rel, relB were detected in MG63 osteosarcoma cells after CXCR4 downregulation followed Akt or LY294002 treatment. (b) Akt enhanced the transcriptional activity of NF- $\kappa B$ promoter. After incubating with or without CXCR4 shRNA followed Akt or LY294002 treatment, cells were prepared for luciferase assays. Values were normalized as the arbitrary luciferase activity. ${ }^{*} \mathrm{P}<0.05$ VS $\mathrm{CXCR} 4$ shRNA treatment, ${ }^{* *} \mathrm{P}<0.01$ VS CXCR4 shRNA treatment.

exposure of osteosarcoma cells to CXCR4 shRNA and LY294002, could remarkably down-regulate the protein level of p-AKT (Fig. 2c). However, the effect of Akt over-

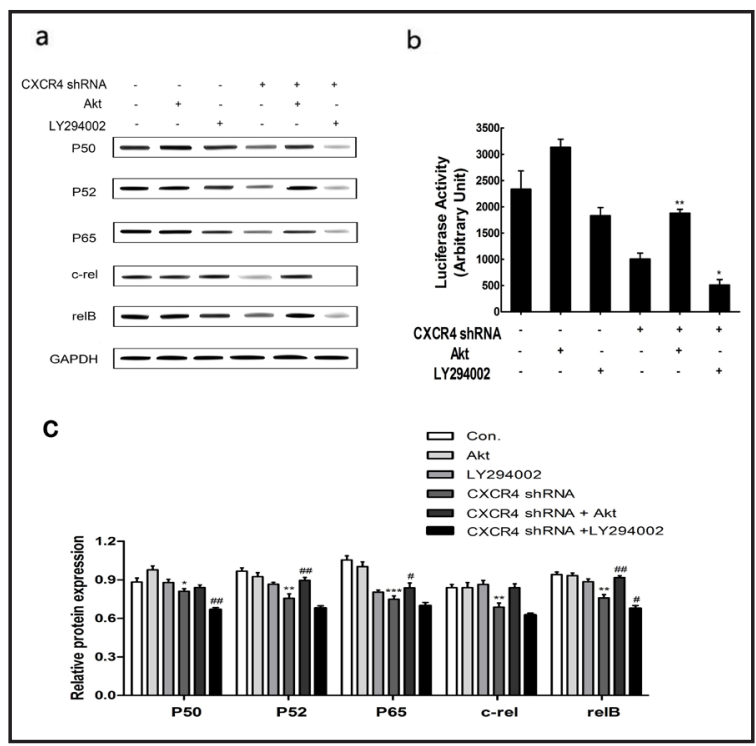
expression and PI3K-AKT inhibitor LY294002 on osteosarcoma cell apoptosis need further study. The results showed treatment with LY29400 could significantly enhance the apoptosis of CXCR4 shRNA (Fig. 2d), whereas treatment with Akt vectors markedly decreased the apoptosis activity of CXCR4 shRNA in osteosarcoma cells (Fig. 2d).

CXCR4 down-regulation induced apoptosis via inhibiting NF- $\kappa \beta$ signaling in osteosarcoma cells

To further elucidate the role of CXCR4 down-regulation in the apoptosis of osteosarcoma cells, we constructed NF- $\kappa \beta$ promoter luciferase reporter plasmid to test whether the activation of NF- $\kappa \beta$ signaling was involved. The results demonstrated that CXCR4 shRNA considerably decreased luciferase expression compared with control in osteosarcoma cells (Fig. 2e). Moreover, the protein expressions of P50, P52, P65, c-rel, relB were obviously decreased in osteosarcoma cells after treatment with CXCR4 shRNA (Fig. 2f). We subsequently investigated the NF- $\kappa \beta$ signal in osteosarcoma cells after treatment with CXCR4 shRNA using IKKa and IkBa vectors. The IKKa protein expression was markedly enhanced in the treatment with CXCR4 shRNA and IKKa vectors compared with CXCR4 shRNA alone treatment (Fig. 2g). In addition, exposure of osteosarcoma cells to CXCR4 shRNA and IkBa vectors, could remarkably promote the protein expression level of IkBa (Fig. $2 \mathrm{~h}$ ). The effect of IKKa and IkBa vectors on osteosarcoma cell apoptosis was further investigated. The results showed treatment with IKKa vector could significantly decrease the apoptosis induced by CXCR4 shRNA (Fig. 2i), whereas treatment with IkBa vectors could markedly increased the apoptosis activity of CXCR4 shRNA in osteosarcoma cells (Fig. 2i).

\section{CXCR4 regulates $N F-\kappa \beta$ signaling through Akt inactivation in osteosarcoma cells}

To investigate the relationship between Akt and NF- $\kappa \beta$ signaling followed CXCR 4 downregulation in osteosarcoma cells, we evaluated the expression of NF- $\kappa \beta$ signaling proteins after CXCR4 down-regulation followed treatment with Akt vectors and PI3K-AKT inhibitor LY294002. The protein expressions of P50, P52, P65, c-rel, relB were detected in osteosarcoma cells after CXCR4 down-regulation followed Akt vectors or LY294002 treatment. The results showed Akt over-expression could promote the protein expressions of P50, P52, P65, c-rel, relB, whereas LY294002 obviously down-regulated the protein expressions of P50, P52, P65, c-rel, relB compared with CXCR4 shRNA treatment alone (Fig. 3a and c). Subsequently, we testified the transcriptional activity of NF- $\kappa \beta$ promoter followed treatment with CXCR4 shRNA and Akt vectors or LY294002. The results demonstrated that Akt over-expression could significantly increase luciferase expression driven by NF- $\kappa \beta$ promoter, whereas 
Fig. 4. CXCR4 down-regulation induced apoptosis involved ERS signaling in osteosarcoma cells. (a) The protein expressions of CHOP, GRP78, XBP-1, P-eIF2a, eIF2a were detected in MG63 osteosarcoma cells after CXCR4 down-regulation. (b) The protein expressions of CHOP, GRP78, XBP-1, P-eIF2a, eIF2a were detected in MG63 osteosarcoma cells after CXCR4 down-regulation followed CHOP, GRP78, XBP-1, P-eIF2a ShRNA treatment. (c, d) The protein expressions of DR5, Caspase-3, Bcl-2, BAX were detected in MG63 osteosarcoma cells after CXCR4 down-regulation followed CHOP, GRP78, XBP-1, P-eIF2a ShRNA treatment. (e) Cell apoptosis was detected for osteosarcoma cells treated with CXCR4 shRNA followed transfection with CHOP, GRP78, XBP1, P-eIF2a ShRNA. ${ }^{*} \mathrm{P}<0.05$ VS CXCR4 shRNA treatment, ${ }^{* *} \mathrm{P}<0.01$ VS Blank. ${ }^{* * *} \mathrm{P}<0.001$ VS CXCR4 shRNA treatment.

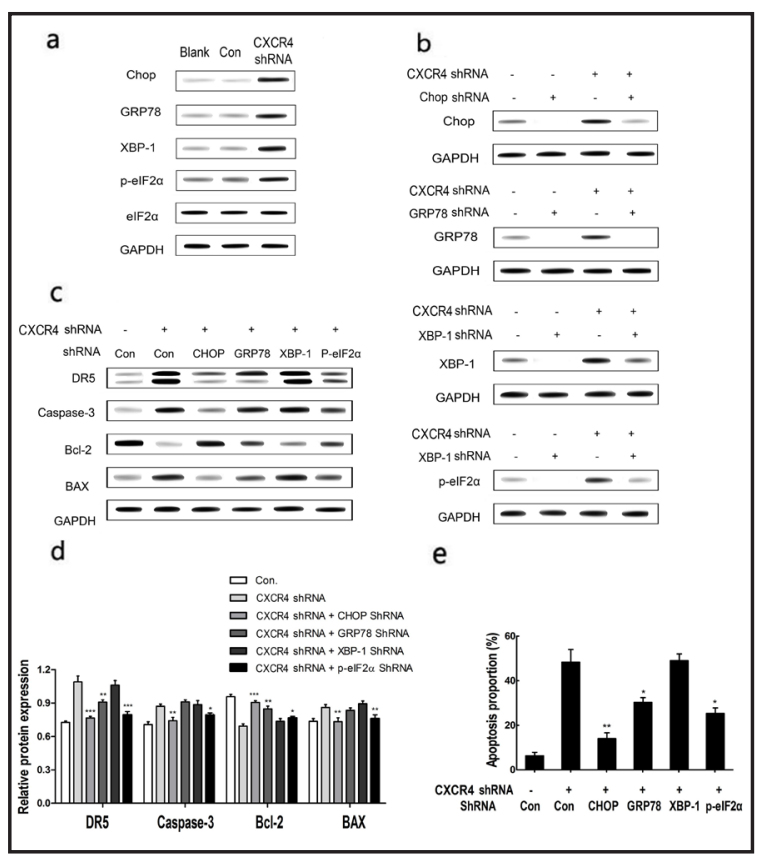

LY294002 markedly decreased the transcriptional activity of NF- $\kappa \beta$ promoter compared with CXCR4 shRNA treatment alone (Fig. 3b).

CXCR4 down-regulation induced apoptosis involved ERS signaling in osteosarcoma cells

Whether apoptosis induced by CXCR4 down-regulation in osteosarcoma cells was associated with ERS signaling need to be further investigated. To detect whether ERS signaling was involved in CXCR4 down-regulation induced apoptosis, the expression of ERS relevant markers including CHOP, GRP78, XBP-1, P-eIF2 $\alpha$, eIF2 $\alpha$ were determined by western blot. The results showed that CHOP, GRP78, XBP-1, P-eIF2 $\alpha$ protein expression levels were significantly increased with exposure to CXCR4 shRNA, while not the eIF2 $\alpha$ (Fig. 4a). Furthermore, we determined the knockdown efficiency of CHOP, GRP78, XBP-1 or P-eIF2 $\alpha$ after treatment with CXCR4 shRNA (Fig. 4b). Interestingly, knockdown of CHOP and P-eIF2 $\alpha$ could downregulate the protein levels of DR5, Caspase-3 and BAX and increased Bcl-2 protein level, while knockdown of GRP78 could remarkably downregulate the protein levels of DR5 and increased Bcl-2 protein level combined with CXCR4 shRNA treatment compared with CXCR4 shRNA treatment alone (Fig 4c and d). Consistently, downregulation of CHOP, GRP78, P-eIF $2 \alpha$ instead of XBP-1 could significantly inhibit apoptosis followed treatment with CXCR4 shRNA compared with CXCR4 shRNA alone treatment (Fig. 4e).

\section{Discussion}

Osteosarcoma oncogenesis is a multistep process that involves multiple process including oncogenes and cell invasion and metastasis, detached from the primary tumor site and adhere to local migration matrix through stromal tissue, which depend on cell communication and cell to matrix interactions [30,31]. Clinical studies demonstrated that CXCR4 was up-regulated in various cancers and involved in cancer cells angiogenesis and metastasis $[32,33]$. Previous studies revealed that CXCR4 inhibitor could induce apoptosis and inhibit proliferation of osteosarcoma cells in vitro [34]. Oda et al. also showed high expression of CXCR4 was detected in metastatic and primary sites, which correlated with activation of vascular endothelial growth factor signaling pathway [35]. However, the detailed mechanisms of how CXCR4 regulating the death of osteosarcoma cells need to be further elucidated, especially the influence on the downstream signaling pathways. 
This study demonstrated that the expression level of CXCR4 in osteosarcoma cells was higher than in normal osteoblast cells. To investigate the effect of CXCR4 on osteosarcoma cells, we found down-regulation of CXCR4 significantly reduced the cell proliferation and remarkably increased the cell apoptosis in osteosarcoma cells. Previous study revealed the enhanced CXCR4 expression was involved in distant metastasis of human osteosarcoma cells [36]. In addition, the CXCR4 blocking antibody eliminated pulmonary lesions from human osteosarcoma cells in a lung-seeding tumor model [37]. Consistently, the apoptotic protein expression levels including PARP, caspase-3, caspase-8, and caspase- 9 were obviously enhanced after CXCR4 shRNA treatment, whereas Bid protein expression level was expectedly decreased with CXCR4 shRNA treatment in osteosarcoma cells. Furthermore, the results demonstrated that down-regulation of CXCR4 induced cell apoptosis was caspase dependent evidenced by z-VAD-fmk, z-IETD-fmk and z-LEHD-fmk caspase inhibitors treatment blocked osteosarcoma cells apoptosis induced by CXCR4 shRNA treatment. Previous study showed that silencing of CXCR4 could induce apoptosis of triple-negative breast cancer cells, involving the promoted protein level of caspase-3 [38]. To further explore the molecular mechanisms of CXCR4 shRNA in osteosarcoma cell apoptosis, PI3K-AKT and NF- $\kappa \beta$ signaling pathway were detected and the results showed that the cell apoptosis of osteosarcoma induced by down-regulation of CXCR4 through inhibition of PI3K-AKT and NF- $\kappa \beta$ signaling pathway. The results showed LY29400 could significantly increase the cell apoptosis induced by down-regulation of CXCR4. However, treatment with Akt vectors could markedly decrease the apoptosis proportion induced by down-regulation of CXCR4 in osteosarcoma cells. Interestingly, similar with LY29400, transfection with IkBa vectors could prominently increase the apoptosis proportion induced by CXCR4 shRNA, whereas treatment with IKKa vectors could significantly decrease the cell apoptosis induced by down-regulation of CXCR4 in osteosarcoma cells. The roles of PI3K-AKT and NF- $\kappa \beta$ in the regulation of cell growth, proliferation and apoptosis had been extensively investigated [39, 40]. Previous studies revealed that PI3K/AKT/NF- $\kappa \beta$ signaling pathway played a vital role in regulating apoptosis in various cancer cells [41-44]. Yu et al. indicated that celastrol induced apoptosis and decreased cell invasion and migration via inhibition of PI3K/AKT/NF- $\kappa \beta$ signaling pathway in human osteosarcoma cells [45]. Thus, we further investigated the relationship between Akt and NF- $\kappa \beta$ signaling followed CXCR4 down-regulation treatment in osteosarcoma cells. As expected, the results indicated that Akt over-expression could promote the activation of NF- $\kappa \beta$, while LY294002 treatment reversed outcome in osteosarcoma cells, which determined by the protein expression levels of P50, P52, P65, c-rel, relB and the transcriptional activity of NF- $\kappa \beta$ promoter. Therefore, our results suggested that downregulation of CXCR4 induced apoptosis via inhibiting PI3K/Akt/NF- $\kappa \beta$ signaling pathway in osteosarcoma cells. In addition, we also investigated whether ERS signaling was involved in down-regulation of CXCR4 induced apoptosis in osteosarcoma cells. The results showed that ERS related protein expression levels were obviously increased with down-regulation of CXCR4. Knockdown of ERS related proteins could significantly inhibit cell apoptosis and the apoptotic protein expression levels induced by the down-regulation of CXCR4. These results suggested that down-regulation of CXCR4 induced apoptosis was involved in ERS activation in osteosarcoma cell.

\section{Conclusion}

These findings showed that down-regulation of CXCR4 induced apoptosis via inhibiting PI3K/Akt/NF- $\kappa \beta$ signaling pathway in osteosarcoma cells. Meanwhile, ERS activation was involved in down-regulation of CXCR4 induced osteosarcoma cell apoptosis. We further demonstrated knockdown of ERS related proteins followed down-regulation of CXCR4 could significantly inhibit cell apoptosis. These findings provided a novel mechanism of osteosarcoma treatment by down-regulation of CXCR4 induced apoptosis through PI3K/ 


\section{Cellular Physiology Cell Physiol Biochem 2018;46:2250-2260 \begin{tabular}{l|l} 
DOI: 10.1159/000489593 & O 2018 The Author(s). Published by S. Karger AG, Basel \\
www.karger.com/cpb
\end{tabular} \\ Jiang et al.: Mechanism of CXCR4 on Osteosarcoma}

Akt/NF- $\kappa \beta$ signaling pathway. This newly mechanism could be a new potential therapeutic manner and improve the outcome of treatment in osteosarcoma.

\section{Acknowledgements}

The work was supported by the National Natural Science Foundation of China (Grants No.: 81472511), Zhejiang Provincial Natural Science Foundation of China (Grant No.: LY16H160044) and the Medicine and Health Foundation of Zhejiang Province (Grant No.: 2015KYB285).

\section{Disclosure Statement}

The author(s) declared no potential conflicts of interest with respect to the research, authorship, and/or publication of this article.

\section{References}

1 Kansara M, Teng MW, Smyth MJ, Thomas DM: Translational biology of osteosarcoma. Nat Rev Cancer 2014;14:722-735.

- Gorlick R, Khanna C: Osteosarcoma. J Bone Miner Res 2010;25:683-691.

3 Ottaviani G, Jaffe N: The epidemiology of osteosarcoma. Cancer Treat Res 2009;152:3-13.

4 Messerschmitt PJ, Garcia RM, Abdul-Karim FW, Greenfield EM, Getty PJ: Osteosarcoma. J Am Acad Orthop Surg 2009;17:515-527.

-5 Kundu ZS: Classification, imaging, biopsy and staging of osteosarcoma. Indian J Orthop 2014;48:238-246.

6 Wan J, Zhang X, Liu T, Zhang X: Strategies and developments of immunotherapies in osteosarcoma. Oncol Lett 2016;11:511-520.

7 Park JA, Shin HY: Influence of genetic polymorphisms in the folate pathway on toxicity after high-dose methotrexate treatment in pediatric osteosarcoma. Blood Res 2016;51:50-57.

-8 Soares PI, Dias SJ, Novo CM, Ferreira IM, Borges JP: Doxorubicin vs. ladirubicin: methods for improving osteosarcoma treatment. Mini Rev Med Chem 2012;12:1239-1249.

$>9$ Wong RS: Apoptosis in cancer: from pathogenesis to treatment. J Exp Clin Cancer Res 2011;30:87.

10 Elmore S: Apoptosis: a review of programmed cell death. Toxicol Pathol 2007;35:495-516.

11 Jin Z, El-Deiry WS: Overview of cell death signaling pathways. Cancer Biol Ther 2005;4:139-163.

12 Baranski Z, Booij TH, Kuijjer ML, de Jong Y, Cleton-Jansen AM, Price LS, van de Water B, Bovée JV, Hogendoorn PC, Danen EH: MEK inhibition induces apoptosis in osteosarcoma cells with constitutive ERK1/2 phosphorylation. Gene Canc 2015;6:503-512.

13 Hoesel B, Schmid JA: The complexity of NF-kappaB signaling in inflammation and cancer. Mol Cancer 2013;12:86.

14 Li F, Zhang J, Arfuso F, Chinnathambi A, Zayed ME, Alharbi SA, Kumar AP, Ahn KS, Sethi G: NF-kappaB in cancer therapy. Arch Toxicol 2015;89:711-731.

15 Israel A: The IKK complex, a central regulator of NF-kappaB activation. Cold Spring Harb Perspect Biol 2010;2:a000158.

16 Karin M: How NF-kappaB is activated: the role of the IkappaB kinase (IKK) complex. Oncogene 1999;18:6867-6874.

17 Mundi PS, Sachdev J, McCourt C, Kalinsky K: AKT in cancer: new molecular insights and advances in drug development. Br J Clin Pharmacol 2016;82:943-956.

18 Chen H, Huang Y, Huang J, Lin L, Wei G: Gigantol attenuates the proliferation of human liver cancer HepG2 cells through the PI3K/Akt/NF-kappaB signaling pathway. Oncol Rep 2017;37:865-870.

19 Zhang LL, Mu GG, Ding QSL, Y X, Shi YB, Dai JF, Yu HG: Phosphatase and Tensin Homolog (PTEN) Represses Colon Cancer Progression through Inhibiting Paxillin Transcription via PI3K/AKT/NF-kappaB Pathway. J Biol Chem 2015;290:15018-15029. 


\section{Cellular Physiology Cell Physiol Biochem 2018;46:2250-2260 \begin{tabular}{l|l} 
and Biochemistry Published onlIne: IVlay 07, 2018 & $\begin{array}{l}\text { (c) } 2018 \text { The Author(s). Published by S. Karger AG, Basel } \\
\text { www.karger.com/cpb }\end{array}$
\end{tabular}}

Jiang et al.: Mechanism of CXCR4 on Osteosarcoma

20 Hao NB, Tang B, Wang GZ, Xie R, Hu CJ, Wang SM, Wu YY, Liu E, Xie X, Yang SM: Hepatocyte growth factor (HGF) upregulates heparanase expression via the PI3K/Akt/NF-kappaB signaling pathway for gastric cancer metastasis. Cancer Lett 2015;361:57-66.

21 Pozzobon T, Goldoni G, Viola A, Molon B: CXCR4 signaling in health and disease. Immunol Lett 2016;177:615.

-22 Sun X, Cheng G, Hao M, Zheng J, Zhou X, Zhang J, Taichman RS, Pienta KJ, Wang J: CXCL12 / CXCR4 / CXCR7 chemokine axis and cancer progression. Cancer Metast Rev 2010;29:709-722.

23 Bachelerie F. CXCL12/CXCR4-axis dysfunctions: Markers of the rare immunodeficiency disorder WHIM syndrome. Dis Markers 2010;29:189-198.

24 Neklyudova O, Arlt MJ, Brennecke P, Thelen M, Gvozdenovic A, Kuzmanov A, Robl B, Botter SM, Born W, Fuchs B: Altered CXCL12 expression reveals a dual role of CXCR4 in osteosarcoma primary tumor growth and metastasis. J Cancer Res Clin Oncol 2016;142:1739-1750.

25 Chen G, Wang Z, Liu XY, Liu FY: High-level CXCR4 expression correlates with brain-specific metastasis of non-small cell lung cancer. World J Surg 2011;35:56-61.

26 Hung CS, Su HY, Liang HH, Lai CW, Chang YC, Ho YS, Wu CH, Ho JD, Wei PL, Chang YJ: High-level expression of CXCR4 in breast cancer is associated with early distant and bone metastases. Tumour Biol 2014;35:1581-1588.

27 Feys L, Descamps B, Vanhove C, Vral A, Veldeman L, Vermeulen S, De Wagter C, Bracke M, De Wever O: Radiation-induced lung damage promotes breast cancer lung-metastasis through CXCR4 signaling. Oncotarget. 2015;6:26615-26632.

28 Ren Z, Liang S, Yang J, Han X, Shan L, Wang B, Mu T, Zhang Y, Yang X, Xiong S, Wang G: Coexpression of CXCR4 and MMP9 predicts lung metastasis and poor prognosis in resected osteosarcoma. Tumour Biol 2016;37:5089-5096.

29 Choi WT, Yang Y, Xu Y, An J: Targeting chemokine receptor CXCR4 for treatment of HIV-1 infection, tumor progression, and metastasis. Curr Top Med Chem 2014;14:1574-1589.

30 Broadhead ML, Clark JC, Myers DE, Dass CR, Choong PF: The molecular pathogenesis of osteosarcoma: a review. Sarcoma 2011;2011:959248.

-31 Alfranca A, Martinez-Cruzado L, Tornin J, Abarrategi A, Amaral T, de Alava E, Menendez P, GarciaCastro J, Rodriguez R: Bone microenvironment signals in osteosarcoma development. Cell Mol Life Sci 2015;72:3097-3113.

32 Perissinotto E, Cavalloni G, Leone F, Fonsato V, Mitola S, Grignani G, Surrenti N, Sangiolo D, Bussolino F, Piacibello W, Aglietta M: Involvement of chemokine receptor 4/stromal cell-derived factor 1 system during osteosarcoma tumor progression. Clin Cancer Res 2005;11:490-497.

-33 Katkoori VR, Basson MD, Bond VC, Manne U, Bumpers HL: Nef-M1, a peptide antagonist of CXCR4, inhibits tumor angiogenesis and epithelialtomesenchymal transition in colon and breast cancers. Oncotarget 2015;6:27763-27777.

34 Jiang C, Fang X, Zhang H, Wang X, Li M, Jiang W, Tian F, Zhu L, Bian Z: AMD3100 combined with triptolide inhibit proliferation, invasion and metastasis and induce apoptosis of human U2OS osteosarcoma cells. Biomed Pharmacother 2017;86:677-685.

-35 Oda Y, Yamamoto H, Tamiya S, Matsuda S, Tanaka K, Yokoyama R, Iwamoto Y, Tsuneyoshi M: Author information CXCR4 and VEGF expression in the primary site and the metastatic site of human osteosarcoma: analysis within a group of patients, all of whom developed lung metastasis. Mod Pathol 2006;19:738-745.

-36 Guo M, Cai C, Zhao G, Qiu X, Zhao H, Ma Q, Tian L, Li X, Hu Y, Liao B, Ma B, Fan Q: Hypoxia promotes migration and induces CXCR4 expression via HIF-1 $\alpha$ activation in human osteosarcoma. PLoS One 2014;9:e90518.

-37 Kularatne SA, Deshmukh V, Ma J, Tardif V, Lim RK, Pugh HM, Sun Y, Manibusan A, Sellers AJ, Barnett RS, Srinagesh S, Forsyth JS, Hassenpflug W, Tian F, Javahishvili T, Felding-Habermann B, Lawson BR, Kazane SA, Schultz PG: A CXCR4-targeted site-specific antibody-drug conjugate. Angew Chem Int Ed Engl 2014;53:11863-11867.

38 Liang S, Peng X, Li X, Yang P, Xie L, Li Y, Du C, Zhang G: Silencing of CXCR4 sensitizes triple-negative breast cancer cells to cisplatin. Oncotarget. 2015;6:1020-1030.

-39 Liu Y, Mei C, Sun L, Li X, Liu M, Wang L, Li Z, Yin P, Zhao C, Shi Y, Qiu S, Fan J, Zha X: The PI3K-Akt pathway regulates calpain 6 expression, proliferation, and apoptosis. Cell Signal 2011;23:827-836. 
Jiang et al.: Mechanism of CXCR4 on Osteosarcoma

40 De Castro Barbosa ML, da Conceicao RA, Fraga AG, Camarinha BD, de Carvalho Silva GC, Lima AG, Cardoso EA, de Oliveira Freitas Lione V: NF-kappaB Signaling Pathway Inhibitors as Anticancer Drug Candidates. Anticancer Agents Med Chem 2017;17:483-490.

41 Li J, Liang X, Yang X: Ursolic acid inhibits growth and induces apoptosis in gemcitabine-resistant human pancreatic cancer via the JNK and PI3K/Akt/NF-kappaB pathways. Oncol Rep 2012;28:501-510.

42 Song L, Xiong H, Li J, Liao W, Wang L, Wu J, Li M: Sphingosine kinase-1 enhances resistance to apoptosis through activation of PI3K/Akt/NF-kappaB pathway in human non-small cell lung cancer. Clin Cancer Res 2011;17:1839-1849.

-43 Chen H, Huang Y, Huang J, Lin L, Wei G: Gigantol attenuates the proliferation of human liver cancer HepG2 cells through the PI3K/Akt/NF-kappaB signaling pathway. Oncol Rep 2017;37:865-870.

44 Yu M, Qi B, Xiaoxiang W, Xu J, Liu X: Baicalein increases cisplatin sensitivity of A549 lung adenocarcinoma cells via PI3K/Akt/NF-kappaB pathway. Biomed Pharmacother 2017;90:677-685.

45 Yu X, Wang Q, Zhou X, Fu C, Cheng M, Guo R, Liu H, Zhang B, Dai M: Celastrol negatively regulates cell invasion and migration ability of human osteosarcoma via downregulation of the PI3K/Akt/NF-kappaB signaling pathway in vitro. Oncol Lett 2016;12:3423-3428. 